\title{
HOW DOES ENERgY INTAKE INFLUENCE THE LEVELS OF CERTAIN STEROIDS?
}

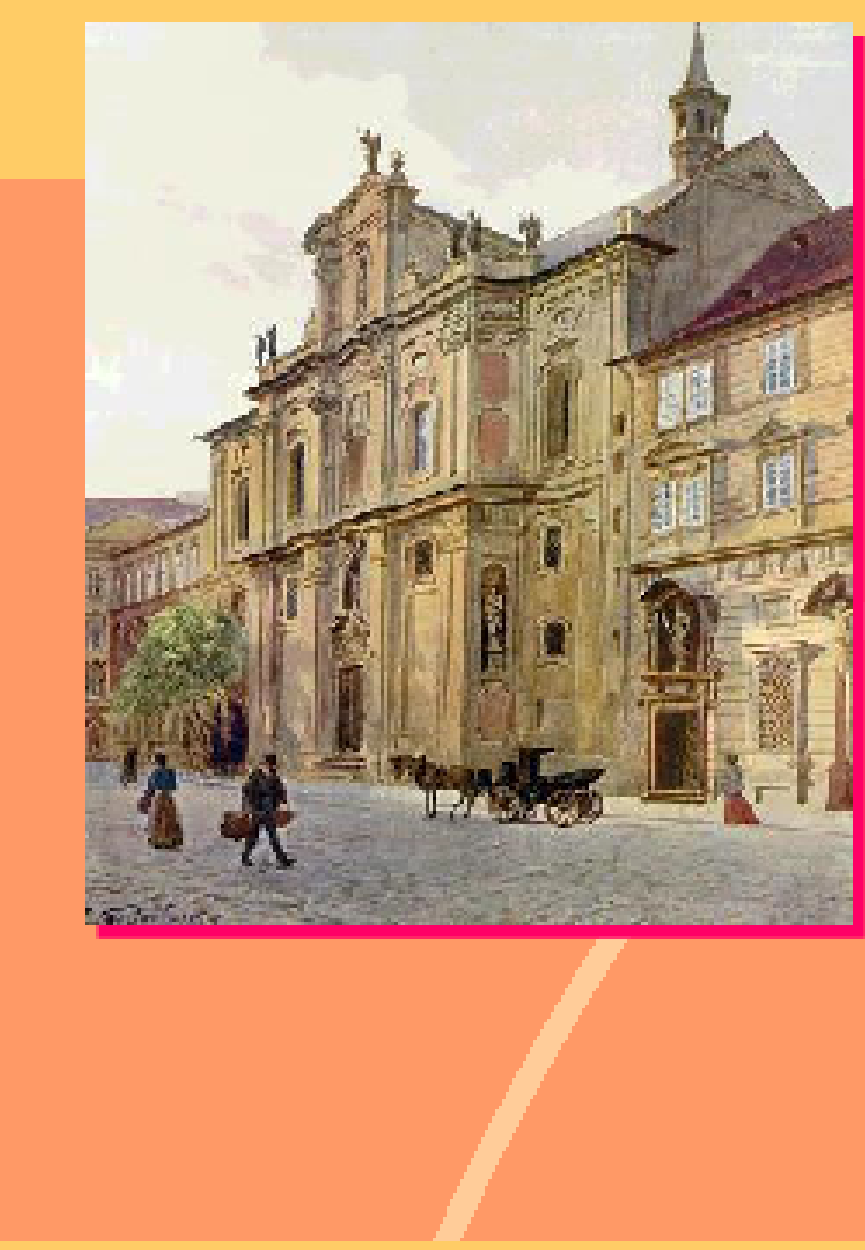

\section{Hana Jandíková, Beáta Rácz, Michaela Dušková, Martin Hill , Karel Vondra, Luboslav Stárka}

Institute of Endocrinology, Prague

Even though the daily profiles of the main sex hormones are well known, small oscillations in their levels that may be connected to food intake have yet to be systematically studied. Glucocorticoids have many various functions in organisms, pituitay (HPA) axis encures tissue and organ-specific reactions of the organism to endogenous and exogenous stimuli (Lightman et al. 2008).

According to a study by Stárka et al. (2015), levels of DHEA and of its conjugated form androstenediol and 7a-hydroxy DHEA decline one to two hours after lunch and dinner. Another recent study has described significant changes of estradiol

The influence of steroid hormones on the food intake is well known. In contrast, however, there are only a few published the food intake is well known.

Prague

\section{Methods}

The aim of this study: Analyse the influence of food intake on steroid hormone and melatonin levels.

As stimuli we selected a standard breakfast, oral and intravenous glucose, and psyllium (as a model of mechanical effects of the food on the gastrointestinal tract).

The study participated eight women with average age of $29.48 \pm 2.99$ years and BMl of $21.3 \pm 1.3 \mathrm{~kg} / \mathrm{m} 2$. All of the women were pre-menopausal, they were non-smokers, healthy, and they were not using any medication or hormonal contraceptive. Blood samples were collected during the The study participated eight women with average age of $29.48 \pm 2.99$ years and $\mathrm{BMI}$ of $21.3 \pm 1.3 \mathrm{~kg} / \mathrm{m} 2$. All of the women were pre-menopausal, they were non-smokers, healthy, and thee
follicular phase (days $1-7$ of the menstrual cycle). Five days before they had undergoing the tests, all of the women followed a standard protocol that did not vary much significantly from the
estst they were informed about the study y rotococoland they signed an informed consent form. The study was approved by the ethical commission of the Institute of Endocrinology in Prague.

OGTT - a oral glucos tolerance test - 75 g of glucose (Glukopur brand) in $250 \mathrm{ml}$ of unsweetened tee perorally.

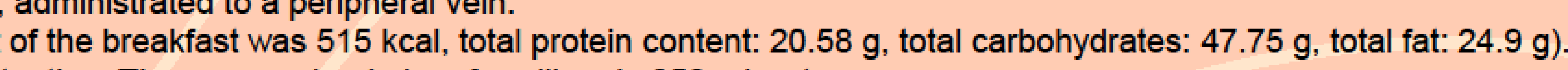

4) psyllium - a non-caloric fibre, which was meant to simulate mechanical stimulation of the gastrointestinal tract through distention. The women drank $4 \mathrm{~g}$ of psyllium in $250 \mathrm{ml}$ water.

- the first sampling was performed at $7: 30$ a.m. after overnight fasting

subsequent samplings were performed at $20,40,60,90$, and 120 minutes

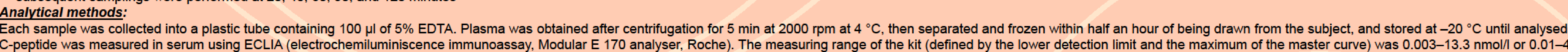
and inter-assay coefficients of variation were $1.5 \%$ and $2.3 \%$, respectively. Blood glucose was measured using the enzymatic reference method with hexokinase (Cobas Integra 400 plus analyser, Roche). The measuring range of the kit was $0.12-40 \mathrm{mmol} /(2.16-720 \mathrm{mg} / \mathrm{dll})$. Intra-set and inter-set reproducibility were $1.7 \%$ and $2.6 \%$, respectively. Cortisol was measured using an RIA kit (Immunotech, France).

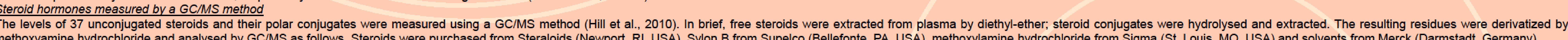
Statistical data analysis:

The changes of steroid levels and melatonin were evaluated using a repeated measures ANOVA model consisting of a Subject factor, explaining differences between subjects, and a Stage factor. Due to the non-Gaussian data distribution and non-constant variance, the original data were

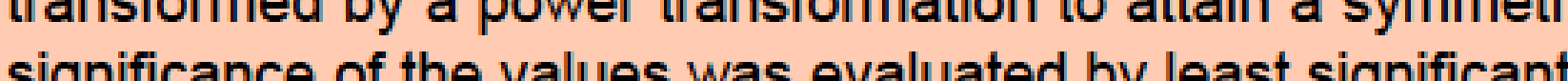

\section{Resulits}
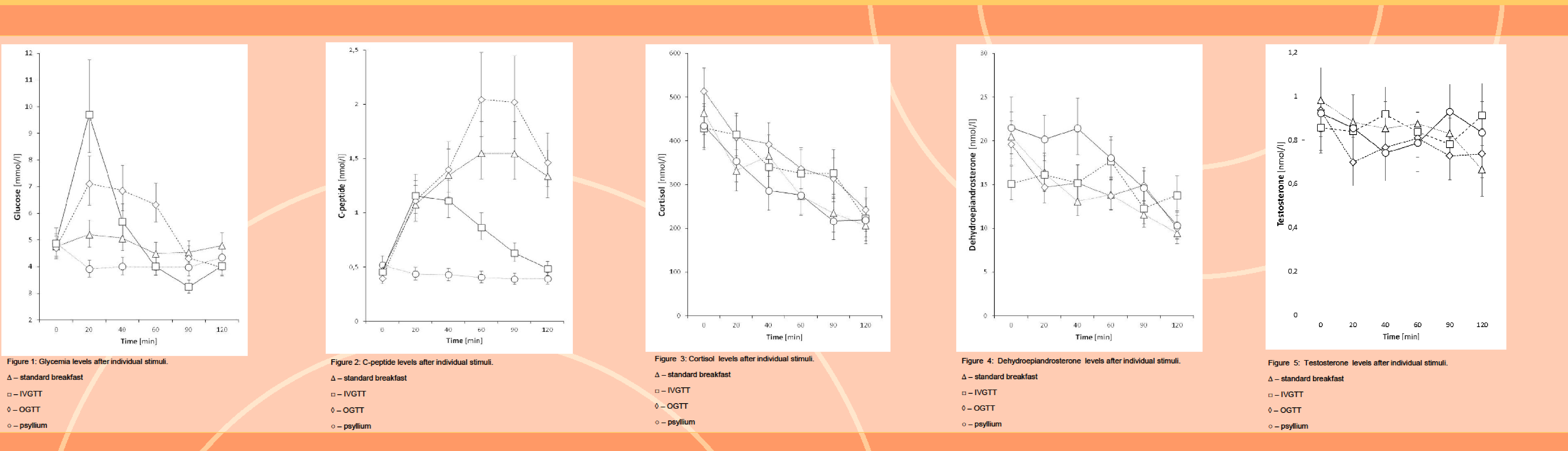

In order to better elucidate various findings on the influence of food intake on hormone levels, we studied the influence of several stimuli on the course of hormone levels. As stimuli we chose a standard

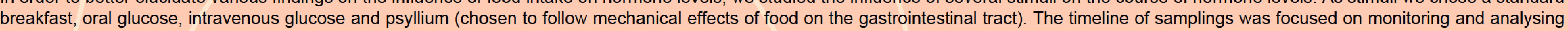
of acute and small changes of hormone levels after each stimulus. As we expected, the glycemia and C-peptide levels reflected normal values of a healthy population (Figures 1 and 2 ).

Cortisol

There was a slowing of the physiological decline in cortisol levels after each of the stimuli, excepting psyllium. This slowing was most pronounced after intravenous glucose, lasting even 60 minutes. After oral glucose and intravenous glucose there was a plateau in cortisol levels, but after breakfast there was an increase in cortisol at 40th minute (Figure 3 ).

DHEA

After the initial decline there was an increase in DHEA after all stimuli. This increase was most pronounced after intravenous glucose, but this increase was delayed compared to the other stimuli (Figure 4).

\section{Testosterone}

The course of testosterone levels did not have any significant relationship to any of the individual stimuli (Figure 5).

Similarly, non-conjugated and conjugated steroids also showed no relationships to individual stimuli, and we were unable to demonstrate a relationship between melatonin and the steroids studied.

\section{Conclusions}

Despite the fact that we performed the tests in the morning hours, when steroid hormone levels physiologically start to change due to their diurnal rhythm, we still found that food intake influences some of the hormone levels. 